\title{
Complex outcome of reciprocal exchange of nuclear DNA between two members of the basidiomycete genus Stereum
}

\author{
A. Martyn Ainsworth, $\dagger$ John R. Beeching, S. John Broxholme, Bridget A. Hunt, \\ Alan D. M. RAYNER* and P. T. SCARD
}

School of Biological Sciences, University of Bath, Claverton Down, Bath BA2 7AY, UK

(Received 6 January 1992; revised 3 February 1992; accepted 6 February 1992)

\begin{abstract}
Strains of the basidiomycete fungi Stereum hirsutum from the USSR and Stereum complicatum from the eastern USA were interfertile, yielding viable, variable, meiotic progeny. However, interactions between paired homokaryotic mycelia of these species were unstable, yielding a uniform mycelial mat on the $S$. complicatum side, but a macroscopically heterogeneous mycelium on the $S$. hirsutum side. Hyphal tips excised from the interactions gave rise to colonies identifiable as one or other of the progenitor strains or two non-progenitor types (C-types and H-types). C-types were all morphologically similar to one another and resembled the $S$. complicatum progenitor except in being inaccessible to nuclear migration and in exhibiting a light-induced phenotypic switch. They could be isolated from both sides of interactions and were somatically compatible with one another. H-types were only isolated from the $S$. hirsutum side of interactions and had a range of morphologies, generally similar to the S. hirsutum progenitor. Pairings between H-types, and between H-types and C-types sometimes resulted in pigmented interaction zones due to somatic incompatibility. Single, dual and minisatellite DNA probes were used to investigate restriction fragment length polymorphisms in progenitor and derivative strains. Nuclear, but not mitochondrial DNA from both species was detected in all non-progenitor strains obtained from beyond the interaction interface. However, whereas $S$. complicatum-specific nuclear sequences were consistently present, the distribution of $S$. hirsutum-specific sequences varied, and was partly correlated with possession of $\mathbf{H}$-type morphologies.
\end{abstract}

\section{Introduction}

Mating in eukaryotes results in the temporary or persistent co-existence of different sets of genetic information (genomes) within the same cell or cell line. Any disparity in the information contained in the associated genomes could lead to conflict and competition between them, and hence to considerable instability and variability in their relationships. An important issue therefore concerns the extent to which distinctive genomes brought together by mating can reconcile and/or benefit from their differences, and hence co-exist stably (Rayner, 1991). By the same token, if they cannot co-exist stably, what may be the consequences in terms of

\footnotetext{
* Author for correspondence. Tel. (0225) 826826 ext. 5419; fax (0225) 826779.

† Present address: Primrose Cottage, 102 North Road, Combe Down, Bath BA2 5DJ, UK.

Abbreviations: mtDNA, mitochondrial DNA; nDNA, nuclear DNA; RFLP, restriction fragment length polymorphism; MA, malt extract agar.
}

evolutionary degeneration, diversification and speciation, and how may these be manipulated for technological applications?

In many eukaryotes, it is difficult to address these issues directly. For example, in some cases, including many fungi, potentially unstable genomic relationships may be avoided if mating is followed more or less immediately by nuclear fusion and meiosis, such that the life cycle is predominantly haploid. Alternatively, as applies in many higher plants and animals, development follows a single associative event, the fusion of two, genetically unique nuclei in female cytoplasm to form a diploid zygote. The fact that all subsequent cell lineages are derived from the zygote may then restrict the potential for the different haploid nuclear genomes to form variable relationships, both with each other and with cytoplasmic genomic organelles (i.e. mitochondria and chloroplasts), in the same organism.

By contrast, in many basidiomycete fungi, mating allows reciprocal exchange and migration of numerous nuclei, but not mitochondria, between fused homokary- 
otic hyphal systems (e.g. Casselton \& Economou, 1985; Hintz et al., 1988; May \& Taylor, 1988). This exchange culminates in the production of extensive heterokaryotic mycelia containing populations of two types of nuclei with compatible mating-type alleles. The nuclei typically do not fuse until immediately prior to meiosis, which occurs in reproductive fruit bodies (basidiomes). The process of associating two types of nuclei within cytoplasmic backgrounds corresponding with two parents is therefore effectively repeated many times. This provides an opportunity for any potential variability in the outcome of particular genomic associations to be expressed.

Another important feature of these basidiomycete matings is that they appear to necessitate the override (Rayner et al., 1984) of somatic incompatibility systems that can cause a rejection response, evident as a demarcation zone, between genetically distinct mycelia. Such rejection responses in themselves provide an indication of the potential instability of genomic associations in basidiomycete mycelia.

Nonetheless, discussions of genetic relationships within and between basidiomycete populations commonly focus on absolutes. Homokaryotic strains are regarded either as compatible $(+)$ or incompatible $(-)$, and the outcome of a successful mating is effectively assumed to be homogeneous.

A different perspective comes from viewing basidiomycete mycelia as dynamic, heterogeneous systems in which the acceptance of nonself and stable genomic relationships implicit in a compatible mating are delicately counterpoised against the potential for instability and rejection of nonself due to somatic incompatibility (Rayner et al., 1984; Rayner, 1991). From this perspective, the outcome of hyphal fusions between genetically different strains may be capable, particularly as the disparity between genomes is increased, of being variable, locally unpredictable and sensitively dependent on cytoplasmic conditions within the participants.

It is therefore interesting that whereas reciprocal matings within a breeding population usually give rise to a single, stable heterokaryon, matings between geographically or ecologically isolated populations can indeed have more complex and/or unstable outcomes (May, 1988; Ainsworth \& Rayner, 1989; Ainsworth et $a l ., 1990 a, b)$. It is now important to understand the molecular processes underlying such outcomes, and whether these involve genetic or epigenetic mechanisms, or both.

In this article, we describe an analysis of morphogenetic and molecular events associated with reciprocal exchange of nuclear DNA (nDNA) between homokaryons of two taxonomically distinct, but closely related species of Stereum, S. complicatum and $S$. hirsutum. Preliminary observations, concerning some of the morphological and cytological characteristics of interactions between these species were previously reported by Ainsworth \& Rayner (1989).

For both species, the strains used were derived directly, i.e. without selection, inbreeding or mutagenesis, from outcrossing natural populations. In these populations, mating compatibility is controlled by a single, multiallelic, mating-type factor, and both homokaryons and heterokaryons possess hyphae with multinucleate compartments and verticillate (whorled) clamp connections. Genetically distinct heterokaryons, and homokaryons which for various reasons are not competent to mate, generally exhibit strong somatic incompatibility responses when paired (Ainsworth, 1987).

Heterokaryons can be distinguished from homokaryons on the basis of their inaccessibility to nonself nuclei; in other words, when paired with a homokaryotic strain, the nuclei from the latter cannot gain entry to the former. On the other hand, the two different types of nuclei from the heterokaryon can gain access to the homokaryon, thereby generating heterokaryotic sectors containing the resident nuclear type and one or other of the accessing nuclear types (Coates et al., 1981 ; Coates \& Rayner, 1985a). Alternatively, homokaryons and heterokaryons can be distinguished by use of genetic markers such as restriction fragment length polymorphisms (RFLPs) (Beeching et al., 1989).

\section{Methods}

Strains, pairings on plates and cytology. The Stereum strains were obtained and assigned identification codes as described by Ainsworth \& Rayner (1989). Pairings were made by placing mycelial disc inocula, $6 \mathrm{~mm}$ diameter, cut from the edge of actively growing colonies, approximately $1 \mathrm{~cm}$ apart in the centre of $9 \mathrm{~cm}$ or $14 \mathrm{~cm}$ diameter plastic non-vented Petri dishes containing 15 or $50 \mathrm{ml} 2 \%(\mathrm{w} / \mathrm{v})$ malt extract agar (MA; $20 \mathrm{~g}$ Munton \& Fison spray malt A, $20 \mathrm{~g} \mathrm{Lab} \mathrm{M}$ agar no. 2 , per litre of distilled water). The plates were incubated at $25^{\circ} \mathrm{C}$ in darkness for up to 6 weeks.

One combination of strains, designated JG1.5 (S. complicatum) and EP18.7 ( $S$. hirsutum) was selected for detailed analysis. The interaction plates were sampled by excising small pieces of mycelium, about $2-12 \mathrm{~mm}^{3}$, from various spatial locations on interaction plates and either using these directly to give rise to 821 cultures from single hyphal tips (following the method of Butler, 1984), or transferring them to fresh medium to develop into 203 mature colonies. In early experiments, such sampling was done qualitatively, according to observed patterns of interaction, but later a more analytical procedure, dictated by preset time and space intervals, was adopted (see Fig. 1). Some subcultures from interaction plates eventually produced basidiospores. Some of these were used to isolate sets of up to 17 singlebasidiospore derivatives as described by Coates \& Rayner (1985b). Five sets of 100 spores and ten corresponding sets from wild fruit bodies from outcrossing British populations of $S$. hirsutum were stained with the fluorochrome DAPI (4,6-diamidino-2-phenylindole) as described by Hooley et al. (1982), but without fixation. The relative DNA contents of these spores were measured using a Leitz MPV3 
(a)

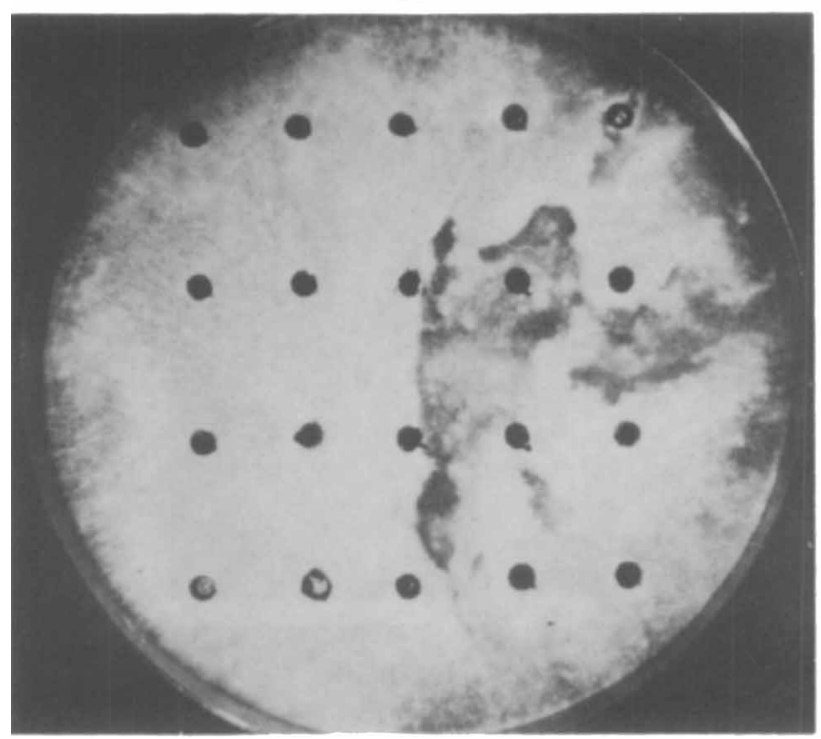

(b)

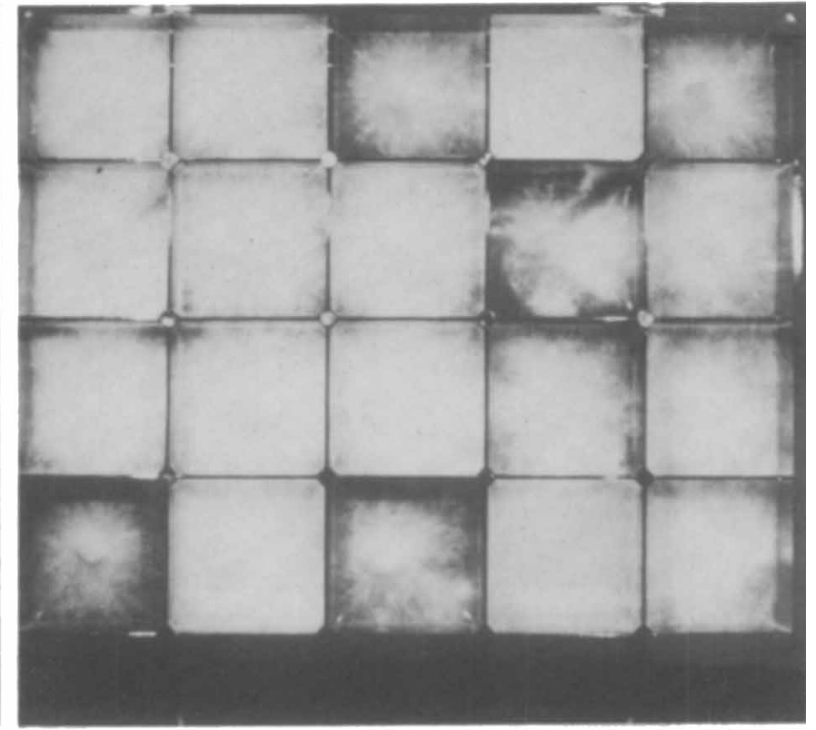

Fig. 1. Quantitative sampling of an interaction between $S$. complicatum (left-hand side of the Petri dish, a), and $S$. hirsutum (right-hand side) paired on a $14 \mathrm{~cm}$ diameter malt agar plate. Sample plugs, $4 \mathrm{~mm}$ diameter were excised in a $5 \times 4$ pattern, at $2 \mathrm{or} 3 \mathrm{~cm}$ intervals respectively $(a)$. The samples were bisected and one half of each was transferred, in the same order as in the pairing, to a $100 \mathrm{~cm}^{2} s q u a r e$ plastic Repli-dish with compartments containing malt agar $(b)$, and the colonies allowed to grow out. Two plates at a time were sampled in this way at weekly intervals up to 5 weeks. The half of each sample not subcultured as shown was used to provide four hyphal tip derivatives as described in the text. One of these derivatives, from alternating sampling points at complementary positions on the two dishes was used for molecular analysis. The phenotypes of all hyphal tip and mass hyphal transfers were recorded.

microspectrofluorometer, with uninucleate basidiospores of Phanerochaete magnoliae (Bath University culture collection) as an internal standard.

Effects of light on phenotypic switching. Progenitor homokaryons, a variety of their siblings, sibling and non-sibling composed heterokaryons of each species and derivative strains from the interactions were inoculated at the centre of $9 \mathrm{~cm}$ diameter Petri plates of $2 \%$ MA. The cultures were incubated at $20^{\circ} \mathrm{C}$ for $15 \mathrm{~d}$ in an incubator with a $12 \mathrm{~h}$ light/dark cycle (light source, two $20 \mathrm{~W}$ Wotan daylight bulbs) and subjected in triplicate to four treatments. In treatment 1 the dishes were wrapped in aluminium foil throughout the incubation period. In treatment 2 they were wrapped for the first $6 \mathrm{~d}$ and then unwrapped for the remaining $9 \mathrm{~d}$. In treatment 3 the dishes were unwrapped for the first $6 \mathrm{~d}$ and then wrapped. In treatment 4 the dishes were left unwrapped throughout.

Colour nomenclature. Colours produced by fungal cultures were identified according to Rayner (1970).

Molecular analysis of interactions. Progenitor and 94 hyphal tipderived isolates from interactions between JG1.5 and EP18.7 were grown in darkness on $8 \mathrm{~cm}$ diameter, sterile, plain, unsoftened, cellulose discs (350 POO, BCL Cellophane, Bridgwater, Somerset), overlying $2 \% \mathrm{MA}$ at $25{ }^{\circ} \mathrm{C}$. The mycelia were harvested, and their total DNA extracted, after 7-9 d as described by Ainsworth et al. $(1990 a, b)$.

Restriction digestion, electrophoresis, Southern transfer and hybridizations were as described in Ainsworth et al. $(1990 a, b)$ with the following exceptions. The M13 minisatellite probe (Brocas et al., 1987) was prepared from the replicative form of M13mp8 (Sigma) as described by Devor et al. (1988), and hybridization with this probe was as in Westneat et al. (1988). In all cases radiolabelling was by the method of Feinberg \& Vogelstein (1983).

\section{Results}

\section{Morphological characteristics}

In replicate pairings of the same combination of $S$. complicatum and $S$. hirsutum strains the interaction patterns were broadly similar, though varied in detail. However, different combinations of strains produced contrasting patterns (Fig. 2). In all cases, flattened zones of mycelium bounded by watery droplets, indicative of access of nonself nuclei (e.g. Ainsworth \& Rayner, 1989), formed in both strains. These were superseded by the development of a uniform mycelial mat, similar to the progenitor, on the $S$. complicatum side of interactions, contrasting with a markedly heterogeneous mycelium on the $S$. hirsutum side.

Hyphal tip derivatives from the interaction between JG1.5 and EP18.7 sometimes resembled, in their morphology and interactive properties, the homokaryotic progenitor on the side from which they were isolated. Alternatively, they were unlike the progenitors in being inaccessible to nuclear migration when paired with homokaryotic tester strains, and prone to become degenerative, with flat, luteous to sienna pigmented mycelia, during subsequent subculturing. These nonprogenitor derivatives increased in frequency as interaction time progressed (Table 1), and were broadly 

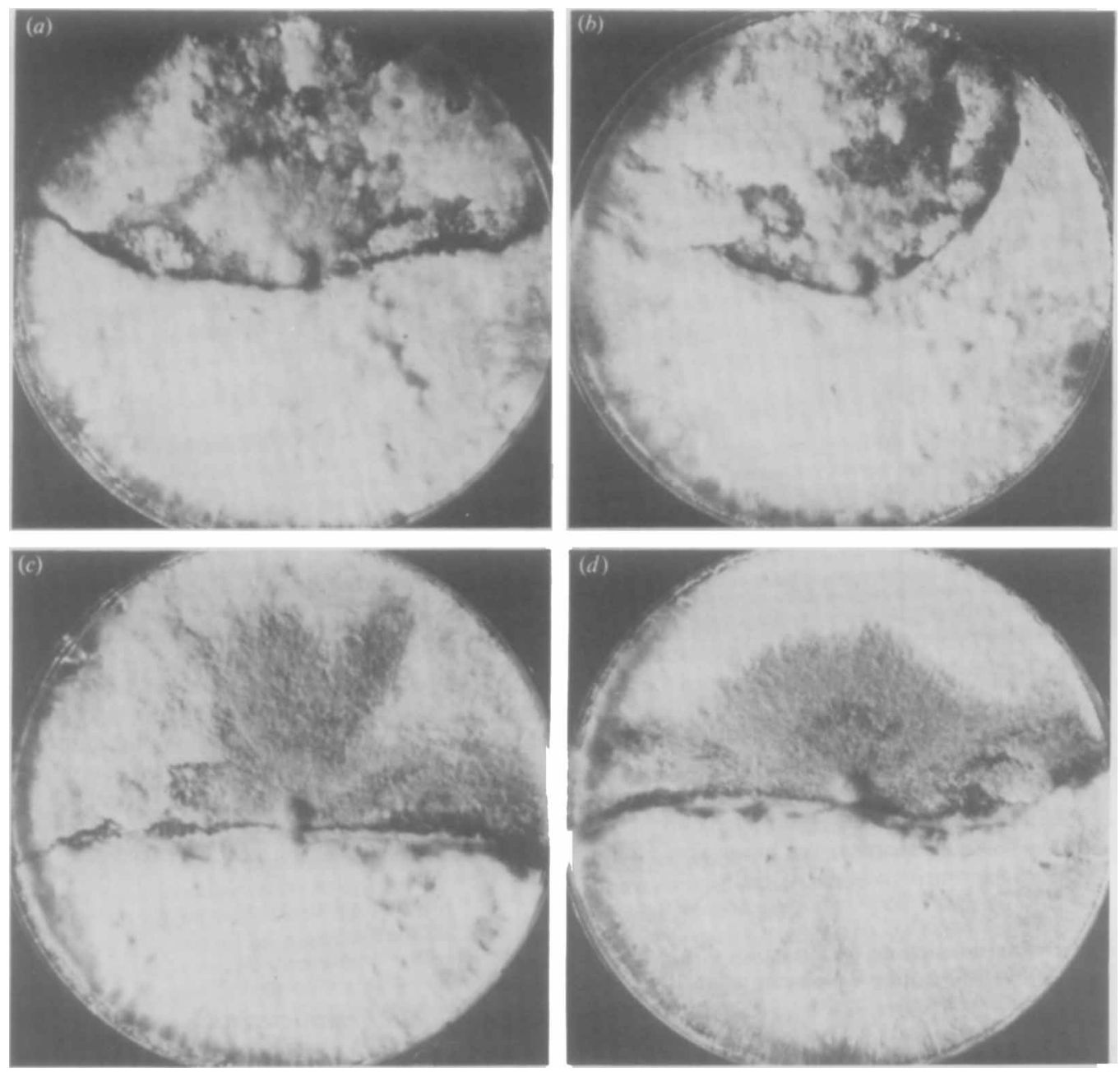

Fig. 2. Variation in interaction pattern between strains of $S$. complicatum and $S$. hirsutum. $(a, b)$ Replicate pairings between strains JG1.5 (S. complicatum, below) and EP18.7 (S. hirsutum, above). $(c, d)$ Replicate pairings between strains JG1.5 (below and EP18.3 (above).

classifiable into two classes, H-types and C-types (Fig. 3). C-types were obtained from both sides of the interactions, but morphologically resembled the $S$. complicatum progenitor. They showed some slight morphological variation, but were all somatically compatible with one another. Many of them exhibited a light-inducible phenotypic switch, producing dense, white, aerial mycelium when grown in darkness, but appressed, luteous to sienna pigmented mycelium when exposed to light/dark cycles (Fig. 4). This switch was not seen in the progenitor, although it was present in some other $S$. complicatum homokaryons.

H-types were only obtained from the $S$. hirsutum side of interactions, resembled the $S$. hirsutum progenitor and lacked the light-induced phenotypic switch of C-types. They comprised a large $(>90 \%)$ group (Hs-types) of isolates that were morphologically similar to one another and a few isolates (Hd-types) which differed, in pigmentation and degree of aerial mycelium production, both from one another and from Hs-types.

Mass hyphal transfers from the $S$. complicatum side of interactions yielded either C-types or pigmented cultures. Similar transfers from the $S$. hirsutum side often yielded colonies with distinctive sectors (Fig. 5).

Pairings between Hs types showed no marked somatic incompatibility, whereas those between Hd-types or between Hs- and Hd-types produced luteous to sienna pigmented interaction zones. The most intense somatic incompatibility reactions were produced when Hd-types were paired with $\mathrm{C}$-types. However, pigmented zones between $\mathrm{Hs}$-types and C-types were either not produced or discontinuous. 
(a)
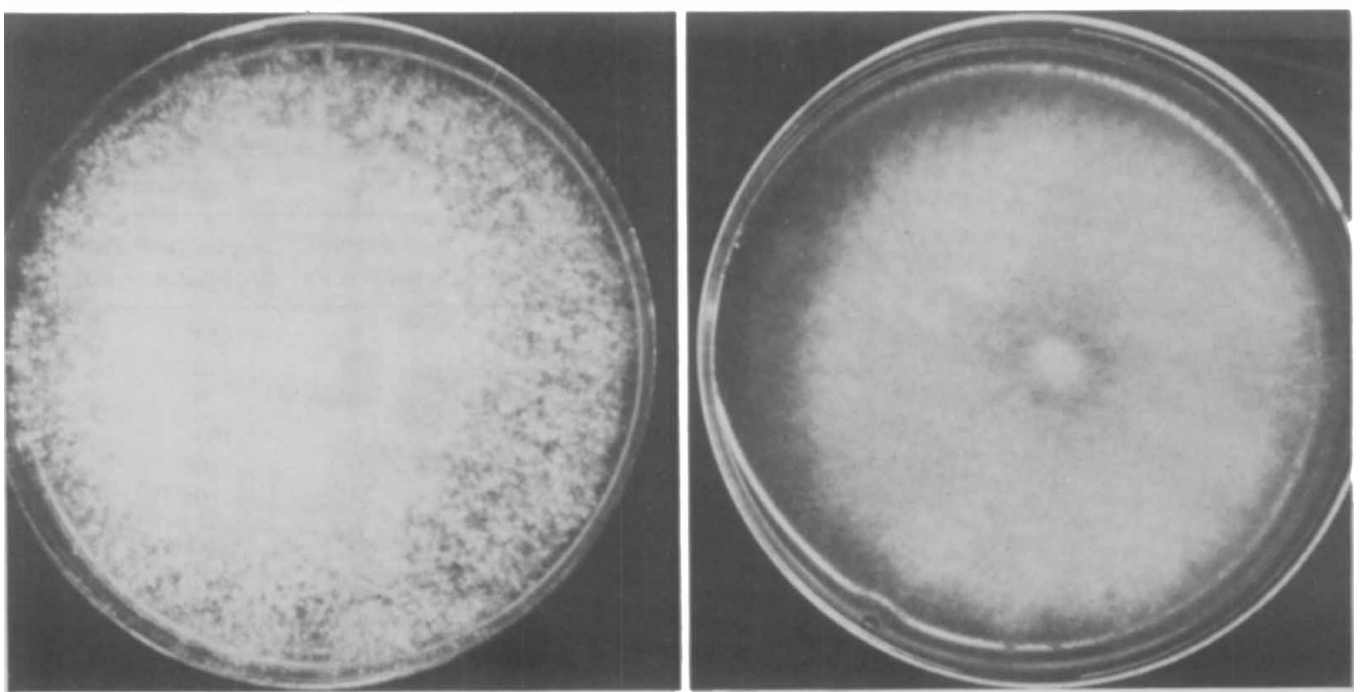

Fig. 3. C-type (a) and H-type (b) non-progenitor colonies derived from hyphal tips isolated from the $S$. hirsutum side of interactions.

(a)

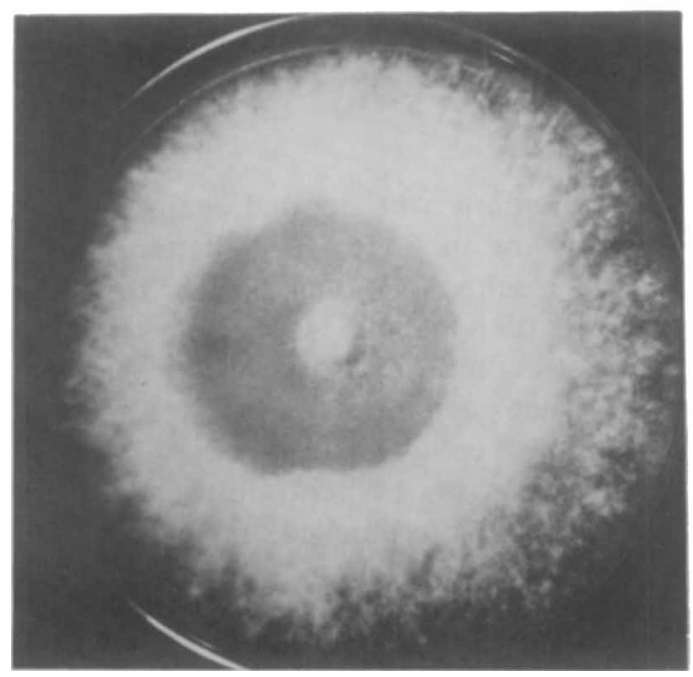

(b)

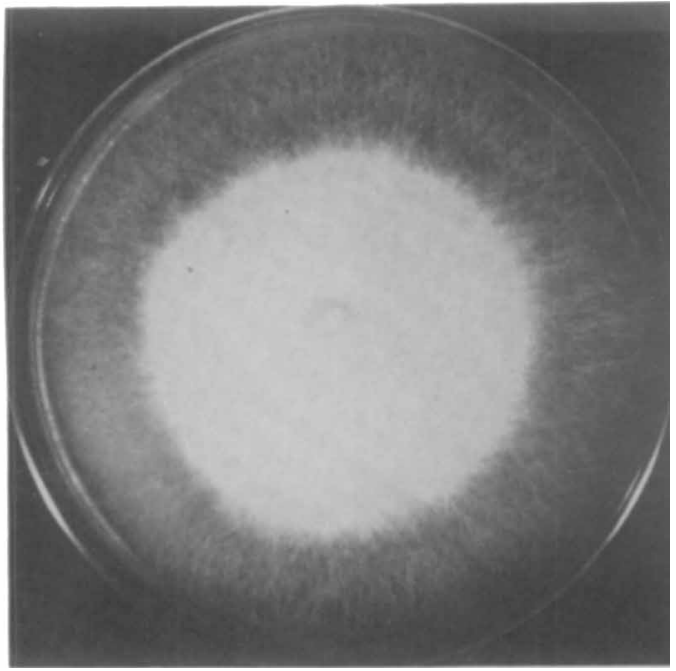

Fig. 4. Response of C-type colonies to changes in illumination. (a) Colony transferred to darkness after initial growth in $12 \mathrm{~h}$ light/dark cycle; $(b)$ colony transferred to light/dark cycle after initial growth in darkness. In both cases an abrupt change in morphology coincides with the time of transfer.

Pairings of C-types and Hs-types against the progenitor strains are illustrated in Fig. 6. Pairings between like phenotypes resulted in production of flattened zones suggesting nuclear access into the progenitors, superseded by establishment of a morphologically uniform mycelial mat. Pairings between Hs-types and the $S$. complicatum progenitor resulted in a luteous to sienna pigmented somatic rejection zone. Pairings between C-types and the $S$. hirsutum progenitor resembled the interaction between the homokaryons of these species, except that invasion of $S$. hirsutum was less extensive.

The mean relative DNA content of basidiospores derived from the interaction [range 235.0 $\pm 3 \cdot 3$ (standard error) to $253.3 \pm 3.9$ arbitrary units] was not significantly different (approximate test of equality of means, Sokal \& Rohlf, 1981) from that of $S$. hirsutum basidiospores from wild British populations (219.0 \pm 3.3 to $296.7 \pm 8.4)$. Single basidiospore-derived progeny from C-types inter- 


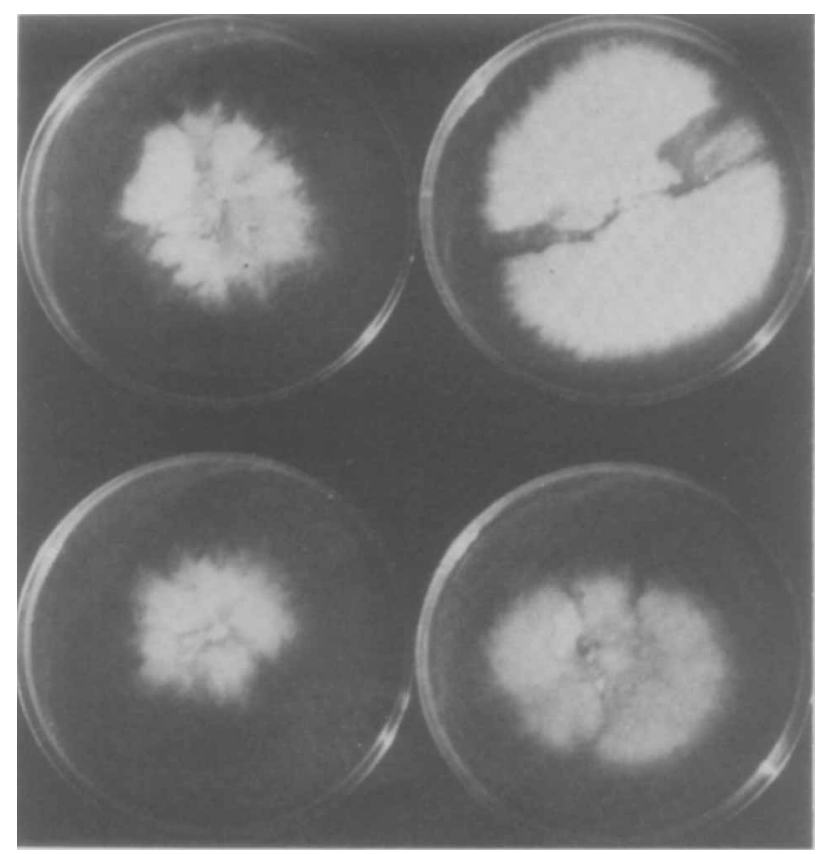

Fig. 5. Colonies formed following mass hyphal transfers from the $S$. hirsutum side of interactions with $S$. complicatum.

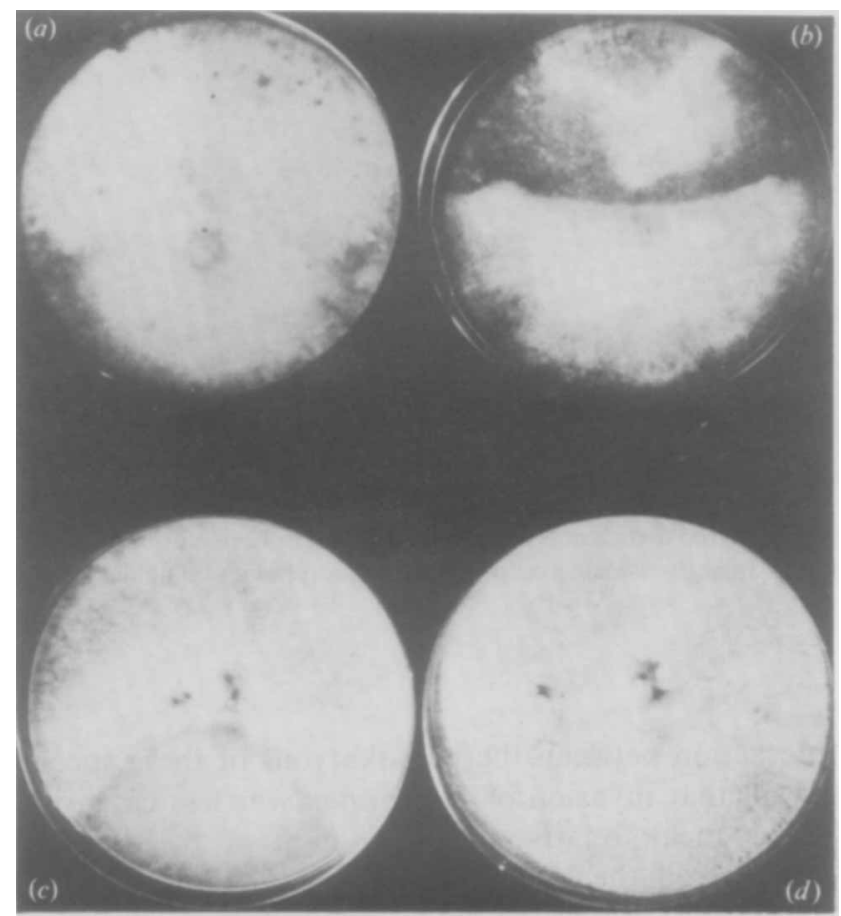

Fig. 6. Pairing of H-type and C-types (lowermost colonies) with progenitor homokaryons (uppermost colonies). (a) H-type vs. S. hirsutum progenitor; (b) C-type vs. S. hirsutum progenitor; (c) H-type vs. $S$. complicatum progenitor; (d) C-type vs. $S$. complicatum progenitor.
Table 1. Phenotypic categories, based on morphological and interactive criteria, assigned to hyphal tip derivatives from quantitatively analysed interactions between $S$. hirsutum and S. complicatum

\begin{tabular}{|c|c|c|c|c|c|}
\hline \multirow[b]{2}{*}{ Week } & \multirow[b]{2}{*}{ Location* } & \multicolumn{4}{|c|}{ Phenotypic categories } \\
\hline & & $\begin{array}{c}\text { hirsutum } \\
\text { progenitor }\end{array}$ & $\begin{array}{l}\text { complicatum } \\
\text { progenitor }\end{array}$ & $\begin{array}{l}\text { H-type non- } \\
\text { progenitor }\end{array}$ & $\begin{array}{l}\text { C-type non- } \\
\text { progenitor }\end{array}$ \\
\hline \multirow[t]{3}{*}{1} & $C$ & $\mathbf{0}$ & 53 & $\mathbf{0}$ & 15 \\
\hline & $I$ & 5 & 0 & 0 & 3 \\
\hline & $\boldsymbol{H}$ & 25 & $\mathbf{0}$ & 3 & 0 \\
\hline \multirow[t]{3}{*}{2} & $C$ & 0 & 103 & 0 & 13 \\
\hline & $I$ & 0 & 0 & 4 & 0 \\
\hline & $H$ & 24 & $\mathbf{0}$ & 15 & 0 \\
\hline \multirow[t]{3}{*}{3} & $C$ & 0 & 47 & 0 & 48 \\
\hline & $I$ & 0 & 0 & 0 & 16 \\
\hline & $\boldsymbol{H}$ & 3 & 0 & 36 & 3 \\
\hline \multirow[t]{3}{*}{4} & $C$ & 0 & 12 & $\mathbf{0}$ & 96 \\
\hline & $I$ & 0 & 0 & 0 & 4 \\
\hline & $H$ & 6 & 0 & 29 & 11 \\
\hline \multirow[t]{3}{*}{5} & $C$ & 0 & 4 & 0 & 96 \\
\hline & $I$ & 0 & 0 & 0 & 8 \\
\hline & $H$ & 0 & 0 & 31 & 19 \\
\hline
\end{tabular}

- $C$, S. complicatum side; $I$, interaction interface; $H, S$. hirsutum side.

acted similarly when paired against progenitor homokaryons and in sibling combinations. The interactions usually resulted in strongly luteous to sienna pigmented confrontation zones. However, non-progenitor types could sometimes be subcultured from near to these zones. It was not possible consistently to allocate the strains to complementary mating types. Second generation progeny from one of the strains were all morphologically identical and intermingled, implying that the strain was homokaryotic.

\section{Molecular events during interactions}

Sample data showing the results of analyses of nDNA and mitochondrial DNA (mtDNA) in colonies derived from single hyphal tips and single basidiospores, and their relation to the origins and phenotypes of some of these derivatives on interaction plates, are provided in Figs 7-9. The data demonstrate the association, in common cytoplasm, of nDNA, but not mtDNA, derived from both species on both sides of the interaction (Figs 7 and 8). Segregation of markers from both progenitors into viable basidiospore derivatives is also shown (Fig. 9).

Data obtained using the minisatellite probe M13 also suggest that the relative contribution of nDNA from the two species in non-progenitor strains was changeable. 


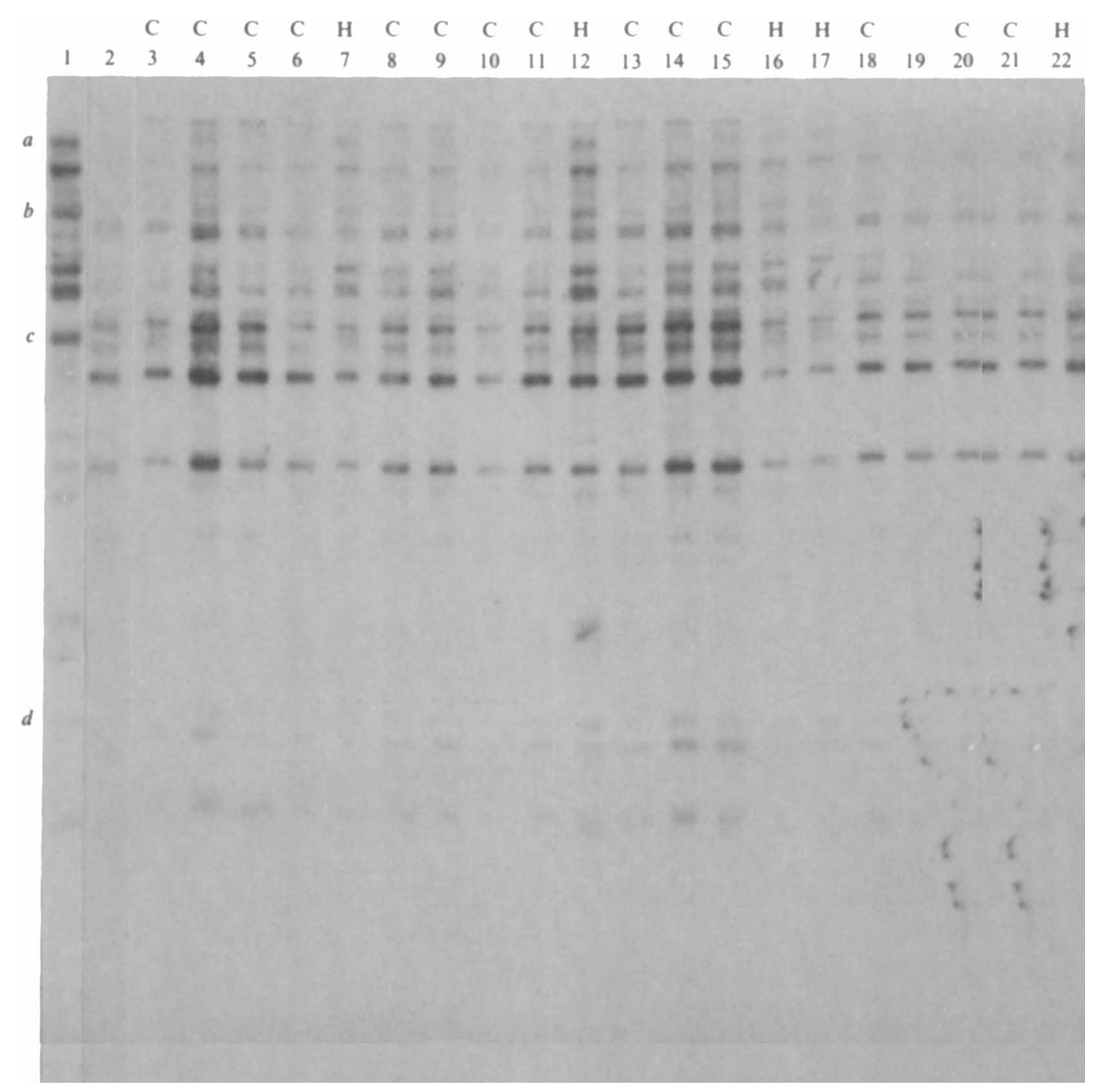

Fig. 7. Comparison of nDNA profiles of the progenitors and hyphal-tip-derived strains from 5-week-old interactions between $S$. complicatum and $S$. hirsutum. DNA, cut with EcoRV, was separated by electrophoresis through a $0.8 \%$ agarose gel in TBE, and blotted and hybridized with an M13-derived minisatellite probe. Tracks 1 and 2 are from the $S$. hirsutum and $S$. complicatum progenitors respectively. Track 19 was from a strain identified phenotypically as a $S$. complicatum progenitor from the $S$. complicatum side. All other tracks are from colonies identified as $\mathrm{H}$ - or $\mathrm{C}$-type non-progenitors (labelled $\mathrm{H}$ or $\mathrm{C}$ respectively). Tracks $3,5,6,10,11,13,18$ and 20 contain only ubiquitous $S$. hirsutum bands (e.g. $b$ and $d$ ); all came from C-types from the $S$. complicatum side. Tracks $7,12,16$ and 17 contains all the $S$. hirsutum-specific bands (e.g. $a, b, c$ and $d$ ); all came from H-types from the $S$. hirsutum side. Tracks 4, 8, 9, 14, 15, 21 and 22 contain some (e.g. $a, b$ and $d$ ) but not all (e.g. $c$ ) of the $S$. hirsutum bands; tracks 9 (C-type) and 22 (H-type) were from the $S$. hirsutum side of interactions whereas the other tracks (all C-types) were from the $S$. complicatum side. No molecular mass markers were included.

Whereas $S$. complicatum-specific bands were detected throughout, irrespective of phenotype, the presence or absence of certain.$S$. hirsutum-specific bands varied. Artificially mixing DNA from the two progenitors in varying proportions prior to hybridization did not reproduce this result.

Some $S$. hirsutum-specific bands were ubiquitous in all derivatives. The possession of others appeared to be hierarchical, such that the derivatives fell into three categories. One category lacked all but the ubiquitous bands, and all the derivatives were C-types from the $S$. complicatum side of interactions (Fig. 7, tracks 3, 5, 6, 10, $11,13,18$ and 20). Another category, consisting purely of H-types, possessed all $S$. hirsutum-specific bands (Fig. 7, 


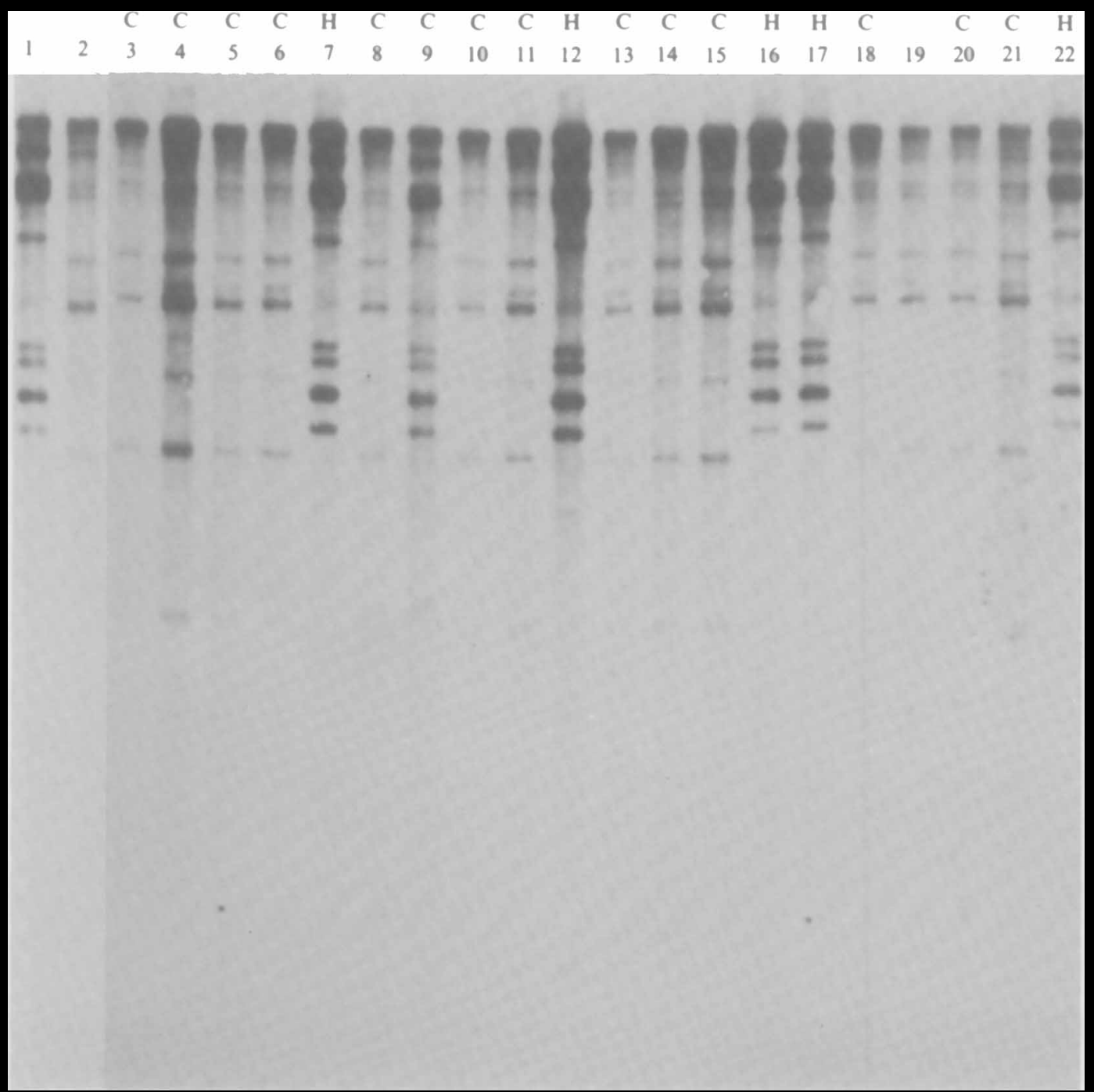




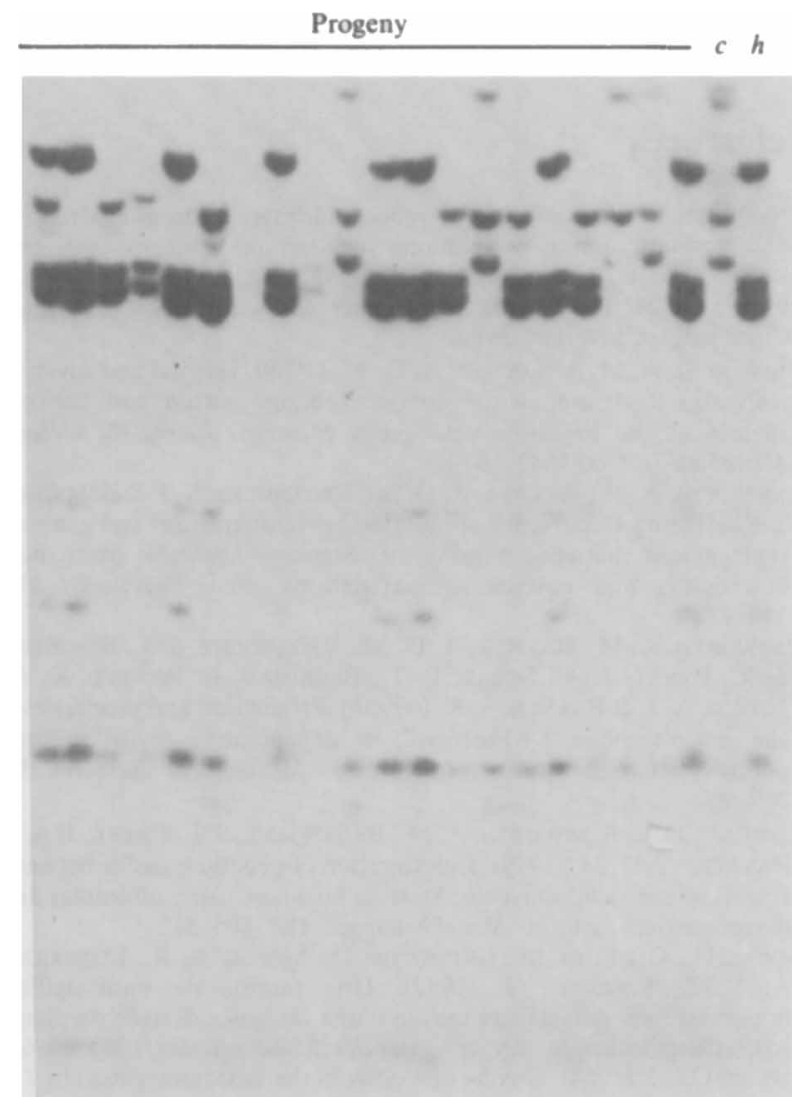

Fig. 9. Sample data of RFLP analysis of single basidiospore progeny from a C-type colony isolated from the $S$. complicatum side of an interaction plate. The probe contained two genomic DNA inserts representing unlinked loci, hence the high proportion of strains showing segregation of RFLP bands contained by $S$. complicatum (track $c$ ) and $S$. hirsutum (track $h$ ) progenitors.

the variation in DNA composition amongst nonprogenitor derivatives revealed by the M13 probe. The relative phenotypic dominance of $S$. complicatum in the interaction is reflected by the consistent presence of the hybridization bands specific to this species in all the derivative strains. On the other hand, the apparent variation in presence of $S$. hirsutum sequences, some of which appeared to be required for an H-type morphology, is consistent with the heterogeneous outcome on the $S$. hirsutum side of interactions. Further work is required in order to understand the origin of this variation and whether it corresponds with any of the known recombinatorial or disruptive mechanisms in fungi. Somatic recombination between nuclei in basidiomycete heterokaryons has been implicated by several studies (e.g. Ellingboe, 1964; Frankel \& Ellingboe, 1977;
Marmeisse, 1991). However, it has usually only been detected under highly selective and restrictive conditions, and the underlying molecular mechanisms are unresolved. The absence of variation in the presence of $S$. complicatum-specific bands in the hybridization profiles would appear to eliminate meiotic or pseudomeiotic recombinatorial mechanisms affecting all chromosomes.

A role for epigenetic mechanisms, based on variation in expression rather than composition of genetic instructions, comes in part from the relatively small genetic differences detected amongst derivative strains. In one case the M13 profiles of two strongly somatically incompatible C- and Hd-types (corresponding with tracks 21 and 22 in Fig. 7) were indistinguishable. Moreover, no major changes in ploidy were detected by examination of the DNA content in dervative basidiospores. Some evidence that genetically identical but phenotypically diverse strains can be generated from protoplast fusions between incompatible strains of the ascomycetous species Trichoderma harzianum has been provided by Stasz et al. (1989). However, here the evidence for genetic identity was indirect, based on expression of isoenzyme polymorphisms rather than DNA composition as such. Another possible parallel, but this time with a prokaryote system, has been found in diploid cells expressing only one progenitor phenotype recovered following protoplast fusion in Bacillus spp. Such non-complementing diploids have been explained in terms of inactivation of one entire genome (Hotchkiss \& Gabor, 1985).

Some indication that the state of expression of nuclear genomes invading from different protoplasmic backgrounds may be crucial in determining phenotypic patterns was provided by the differing outcome between pairings of $\mathrm{C}$ - and $\mathrm{H}$-types with the $S$. hirsutum homokaryotic progenitor. In both cases, the invading nuclear genome would be expected to be derived from $S$. complicatum. However, whereas H-types, where $S$. complicatum nDNA may not be expressed, eventually merged with the $S$. hirsutum progenitor, C-types, where $S$. complicatum nDNA is expressed, largely reproduced the effects of the original interaction between homokaryons. The differing outcome on either side of the original interaction could be explained by supposing that invasive $S$. complicatum nDNA is capable of being expressed in resident $S$. hirsutum protoplasm whereas $S$. hirsutum nDNA, with the possible exception of matingtype sequences, cannot be expressed in $S$. complicatum protoplasm.

Theoretically, resident protoplasm may determine the phenotypic outcome of immigration of nonself $\mathrm{nDNA}$ either on the basis of purely cytoplasmic genetic information or on the basis of nucleo-cytoplasmic 
interactions. The results show that $S$. complicatum nDNA can co-exist, and be expressed, with $S$. hirsutum mtDNA, yielding C-types somatically compatible with similar phenotypes containing $S$. complicatum mtDNA. Differences between the mitochondrial genomes per se cannot therefore explain the observed interaction pattern. On the other hand, there was no evidence that $S$. hirsutum nDNA could co-exist, and be expressed, with $S$. complicatum mtDNA. Moreover, there was no evidence that both $S$. hirsutum and $S$. complicatum nDNA could be co-expressed in the same protoplasmic compartment without giving rise to instability.

Where invading nDNA is expressed, there is a possibility of conflict with pre-existing nuclear-mitochondrial relationships in the resident protoplasm. Such conflict will intensify with genetic difference and may lead to degeneracy and cell death, for example, via activation of aromatic metabolism and associated protease and phenol oxidase enzyme systems. These systems in turn may provide the basis for somatic rejection (Rayner, 1991; Rayner \& Ross, 1991). By avoiding such co-expression, i.e. by suppression of invading or resident genes or genomes, rejection may effectively be overridden (Rayner et al., 1984), but the resulting phenotypic outcome may be complex if there is inconsistency between which genome is suppressed.

Suppression of conflicting nDNA instructions may therefore be an important component of successful mating. In $S$. complicatum-hirsutum interactions we suggest that suppression is incomplete, perhaps because the degree of genetic difference reduces the efficacy of the mating compatibility factors. It is therefore of interest that very similar patterns of phenotypic instability have recently been detected in interactions between homokaryons of $S$. hirsutum with common mating compatibility alleles, but differing at another multi-allelic locus allowing nonself access (A. M. Ainsworth and others, unpublished). These interactions result in a reaction described phenotypically as a 'bowtie' (Coates et al., 1981; Coates \& Rayner, 1985c). The varying phenotypic patterns observed in these interactions can again be explained in terms of varying states of expression of resident and invasive nDNA. The main difference from $S$. complicatum-hirsutum interactions is that some resultant heterokaryons revert readily to homokaryons and allow nuclear access.

The importance of past history in determining states of expression of invasive nuclear genomes in these interactions corresponds with a phenomenon, known as genomic imprinting, whose importance in eukaryote evolutionary processes is increasingly being recognized (Monk \& Surani, 1990). Understanding the underlying mechanisms in fungal systems may be important in future research.
We thank BP Venture Research Unit for financial support.

\section{References}

AINSWORTH, A. M. (1987). Occurrence and interactions of outcrossing and non-outcrossing populations in Stereum, Phanerochaete and Coniophora. In Evolutionary Biology of the Fungi, pp. 285-299. Edited by A. D. M. Rayner, C. M. Brasier \& D. Moore. Cambridge: Cambridge University Press.

AINSWORTH, A. M. \& RAYNeR, A. D. M. (1989). Hyphal and mycelial responses associated with genetic exchange within and between species of the basidiomycete genus Stereum. Journal of General Microbiology 135, 1643-1659.

Ainsworth, A. M., RAYNer, A. D. M., BroXholme, S. J. \& Beeching, J. R. $(1990 a)$. Occurrence of unilateral genetic transfer and genomic replacement between strains of Stereum hirsutum from nonoutcrossing and outcrossing populations. New Phytologist 115, 119-128.

Ainsworth, A. M., Rayner, A. D. M., Broxholme, S. J., Beeching, J. R., Pryke, J. A., Scard, P. T., Berriman, J., Powell, K. A., FloYd, A. J. \& BRANCH, S. K. (1990b). Production and properties of the sesquiterpene, $(+)$-torreyol, in degenerative mycelial interactions between strains of Stereum. Mycological Research 94, 799-809.

Beeching, J. R., Ainsworth, A. M., Broxholme, S. J., Pryke, J. A. \& RAYNER, A. D. M. (1989). Investigation of genetic transfer between strains of the basidiomycete, Stereum hirsutum, using molecular and morphological criteria. New Phytologist 113, 505-512.

Brocas, H., Georges, M., Cristophe, D., Monsieur, R., Lequarre, A. S. \& VASSART, G. (1987). Une famille de minisatellites hypervariables détectée au moyen d'une séquence dérivée du phage M13. Comptes Rendus de l'Academie des Sciences Serie III 304, 67-69.

BUTLER, G. M. (1984). Colony ontogeny in the basidiomycetes. In The Ecology and Physiology of the Fungal Mycelium, pp. 53-71. Edited by D. H. Jennings \& A. D. M. Rayner. Cambridge: Cambridge University Press.

Casselton, L. A. \& Economou, A. (1985). Dikaryon formation. In Developmental Biology of Higher Fungi, pp. 213-229. Edited by D. Moore, L. A. Casselton, D. A. Wood \& J. C. Frankland. Cambridge: Cambridge University Press.

COATES, D. \& RAYNER, A. D. M. (1985a). Heterokaryon-homokaryon interactions in Stereum hirsutum. Transactions of the British Mycological Society 84, 637-645.

COATES, D. \& RAYNeR, A. D. M. (1985b). Genetic control and variation in expression of the 'bow-tie' interaction between homokaryons of Stereum hirsutum. Transactions of the British Mycological Society 84, 191-205.

COATES, D. \& RAYNER, A. D. M. (1985c). Evidence for a cytoplasmically transmissible factor affecting recognition and somato-sexual differentiation in the basidiomycete Stereum hirsutum. Journal of General Microbiology 131, 207-219.

CoAtes, D., RAYNer, A. D. M. \& TodD, N. K. (1981). Mating behaviour, mycelial antagonism and the establishment of individuals in Stereum hirsutum. Transactions of the British Mycological Society 76, 41-51.

Devor, E. J., Ivanovich, A. K., Hickok, J. M. \& Todd, R. D. (1988). A rapid method for confirming cell line identity: DNA 'fingerprinting' with a minisatellite probe from $\mathrm{M} 13$ bacteriophage. BioTechniques 6, 200-202.

Ellingboe, A. H. (1964). Somatic recombination in dikaryon $\mathrm{K}$ of Schizophyllum commune. Genetics 49, 247-251.

FeINBERG, A. \& Vogelstern, B. (1983). A technique for radiolabelling DNA restriction endonuclease fragments to high specific activity. Analytical Biochemistry 132, 6-13.

Frankel, C. \& Ellingboe, A. H. (1977). Sexual incompatibility factors and somatic recombination in Schizophyllum commune. Genetics 85, 427-437.

Hintz, W. E. A., Anderson, J. H. \& Horgen, P. A. (1988). Nuclear migration and mitochondrial inheritance in the mushroom Agaricus bitorquis. Genetics 119, 35-41. 
Hooley, P., Fyfe, A. M., Evola Maltese, C. \& Shaw, D. S. (1982), Duplication cycle in nuclei of germinating zoospores of Phytophthora drechsleri as revealed by DAPI staining. Transactions of the British Mycological Society 79, 563-566.

HotChKISS, R. D. \& GABOR, M. H. (1985). Protoplast fusion in Bacillus and its consequences. In The Molecular Biology of the Bacilli, vol. II, pp. 109-149. Edited by D. A. Dubnau. New York: Academic Press.

MARmeisse, R. (1991). Parasexual analysis in the basidiomycete mushroom Agrocybe aegerita common-B and common $\mathrm{AB}$ crosses. Mycological Research 95, 465-468.

MAY, G. (1988). Somatic incompatibility and individualism in the coprophilous basidiomycete Coprinus cinereus. Transactions of the British Mycological Society 91, 443-451.

MAY, G. \& TAYLOR, J. W. (1988). Patterns of mating and mitochondrial DNA inheritance in the agaric basidiomycete Coprinus cinereus. Genetics 118, 213-220.

Monk, M. \& SURANI, A. (1990). Genomic Imprinting. Cambridge: Company of Biologists.

RAYNER, A. D. M. (1991). The challenge of the individualistic mycelium. Mycologia 83, 48-71.
Rayner, A. D. M., Coates, D., Ainsworth, A. M., Adams, T. J. H., Williams, E. N. D. \& TodD, N. K. (1984). The biological consequences of the individualistic mycelium. In The Ecology and Physiology of the Fungal Mycelium, pp. 509-540. Edited by D. H. Jennings \& A. D. M. Rayner. Cambridge: Cambridge University Press.

RAYNER, A. D. M. \& Ross, I. K. (1991). Sexual politics in the cell. New Scientist 129, 30-33.

RAYNER, R. W. (1970). A Mycological Colour Chart. Kew, Surrey: C.M.I. and British Mycological Society.

SOKAL, R. R. \& ROHLF, F. J. (1981). Biometry, 2nd edn. San Francisco: W. H. Freeman.

Stasz, T. E., Harman, G. E. \& Gullino, M. L. (1989). Limited vegetative incompatibility following intra- and interspecific protoplast fusion in Trichoderma. Experimental Mycology 13, 364-371.

Westneat, D. F., NoOn, W. A., Reeve, H. K. \& Aquadroa, C. F. (1988). Improved hybridization conditions for DNA 'fingerprints' probed with M13. Nucleic Acids Research 16, 4161. 